\title{
Surgical resection of large and giant petroclival meningiomas via a modified anterior transpetrous approach
}

\author{
Xinru Xiao $\cdot$ Liwei Zhang $\cdot$ Zhen Wu - Junting Zhang • \\ Guijun Jia $\cdot$ Jie Tang $\cdot$ Guolu Meng \\ Received: 12 July 2012 /Revised: 22 December 2012 / Accepted: 17 March 2013 / Published online: 18 June 2013 \\ (C) The Author(s) 2013. This article is published with open access at Springerlink.com
}

\begin{abstract}
The authors describe a modified anterior transpetrous approach (ATPA) for the surgical resection of 21 cases of petroclival meningiomas (PCMs). Briefly, a curved periauricular skin incision was used. The cerebellar tentorium and the dura on the petrous apex were coagulated and incised to expose the petrous apex bone fully. The drilling of the petrous apex bone was performed subdurally and began internally from the trigeminal impression, not exceeding $1.5 \mathrm{~cm}$ laterally, not exceeding $6 \mathrm{~mm}$ from the posterior edge of the petrous ridge, and not exceeding $8 \mathrm{~mm}$ in depth from the surface of the petrous bone. The tumors were removed totally in 12 $(57.1 \%)$ cases, subtotally in $8(38.1 \%)$ cases, and partially in $1(4.8 \%)$ case. The transient neurological deficit includes mild oculomotor nerve palsy in three cases, abducens nerve palsy in six cases, language disorder in three cases, and mild hemiplegia in two cases. Facial numbness became worse postoperatively in six patients, and only two patients improved at 6 months after surgery. No death occurred in this series. The modified ATPA is an efficient treatment alterative for large or giant PCMs located at the medial and superior internal acoustic meatus with relatively low risk of complications.
\end{abstract}

Keywords Petroclival - Meningioma - Surgical treatment . Anterior transpetrous approach

\section{Introduction}

Advances in microsurgical techniques, cranial base surgical approaches, and diagnostic neuroimaging methods together

X. Xiao $\cdot$ L. Zhang $(\bowtie) \cdot$ Z. Wu $\cdot$ J. Zhang $\cdot$ G. Jia $\cdot$ J. Tang $\cdot$

G. Meng

Department of Neurosurgery of Beijing Tiantan Hospital, Capital

Medical University, No.6, Tiantan Xili, Dongcheng District,

Beijing 100050, China

e-mail: zhlw2005@163.com

X. Xiao

e-mail: xrttyy2013@163.com with routine intraoperative neurophysiological monitoring have contributed to reduce the morbidity and mortality rates of petroclival meningiomas (PCMs) $[7,19,20,22,24,30]$. However, the permanent neurological deficits and poor functional outcome rates are still relatively high $[18,19,22,30$, 33]. Microsurgical treatment of large and giant PCMs remains a formidable challenge for most neurosurgeons because these tumors frequently adhere tightly to brain stem and encase the cranial nerves, basilar artery, and its perforators. Sufficient exposure of the base of the PCMs is critically important for complete resection of the tumor and protection of adjacent structures. Anterior transpetrous approach (ATPA) is a less aggressive skull base approach and could provide sufficient exposure for the petroclival area and has additional advantages for a selected group of PCMs [12]. Besides, there are also several disadvantages such as troublesome extradural bleeding and the risk of damaging facial nerve and internal carotid artery. In this article, our surgical experience with a simple, modified anterior transpetrous approach is reported.

\section{Patient data and methods}

General data

From June 2009 to June 2011, 21 large or giant PCMs were resected via the modified ATPA at the skull base unit of Beijing Tiantan Hospital. The clinical data of the 21 patients were reviewed retrospectively. They included 5 males and 16 females who ranged in age from 30 to 69 years (mean $50.9 \pm$ 11.1). The course of disease ranged from 2 to 204 months (mean $36.7 \pm 48.8$ months). Of the 21 PCMs, 10 were located in the left side and the remaining 11 were located in the right side. The most common clinical symptoms included headache and dizziness, followed by facial numbness. The occurrence rate of the clinical symptoms and signs is listed in Table 1. 
Table 1 Preoperative symptoms and signs

\begin{tabular}{ll}
\hline Symptom/sign & Number of patients $(n=21)$ \\
\hline Headache, dizziness & $18(85.7 \%)$ \\
Facial numbness & $13(61.9 \%)$ \\
Facial pain & $7(33.3 \%)$ \\
Impaired hearing & $5(23.8 \%)$ \\
Diplopia & $5(23.8 \%)$ \\
Limb weakness & $4(19.0 \%)$ \\
Visual deterioration & $3(14.3 \%)$ \\
Ataxia & $1(4.8 \%)$ \\
Facial palsy & $1(4.8 \%)$ \\
\hline
\end{tabular}

Neuroradiological evaluation

Contrast-enhanced magnetic resonance imaging (MRI) and computed tomography (CT) results were evaluated before surgery for all patients. Thirteen patients $(61.9 \%)$ had radiographic evidence of cavernous sinus (CS) invasion, and nine patients $(42.9 \%)$ exhibited brain stem adherence and edema. There were 3 tumors with their lower margin within upper clivus, 17 extended to the middle clivus, and only 1 case extended to the lower clivus. All of the tumors were confined in the medial side of the internal acoustic meatus (IAM). Tumor size was defined as the greatest contrast-enhancing tumor diameter on MRI or CT scans. The size of the tumors was graded according to the classification of Natarajan et al. [22] as small $(<1.0 \mathrm{~cm})$, medium $(1.0-2.4 \mathrm{~cm})$, large $(2.5-$ $4.4 \mathrm{~cm})$, and giant $(>4.5 \mathrm{~cm})$. In this study, there were 13 cases of giant tumors and 8 cases of large tumors.

Postoperative MRI scans were obtained for the extent of resection determination in each patient within 3 months of surgery (Fig. 1). Tumor removal degree was divided into three grades according to intraoperative observation and postoperative MRI findings: (1) Gross total resection: no tumor residual showed on the immediate postoperative MRI or by the surgeon's observation under the microscope during the operation; (2) subtotal resection: tumor residual less than $10 \%$ of original volume; and (3) partial resection: tumor residual larger than $10 \%$ of original volume. Tumor recurrence was defined as any newly identified enhancement after gross total resection or any increase in tumor volume after near total resection or subtotal resection.

\section{Surgical technique}

The patient was placed in a lateral decubitus position with routine monitoring of cranial nerves III-XII and brain stem auditory evoked potential. The skin incision began $0.5 \mathrm{~cm}$ below the zygomatic arch, $2 \mathrm{~cm}$ anterior to the tragal notch, and extends 10 to $11 \mathrm{~cm}$ superoposteriorly (Fig. 2a). The skin over the zygomatic arch was incised, and the temporalis

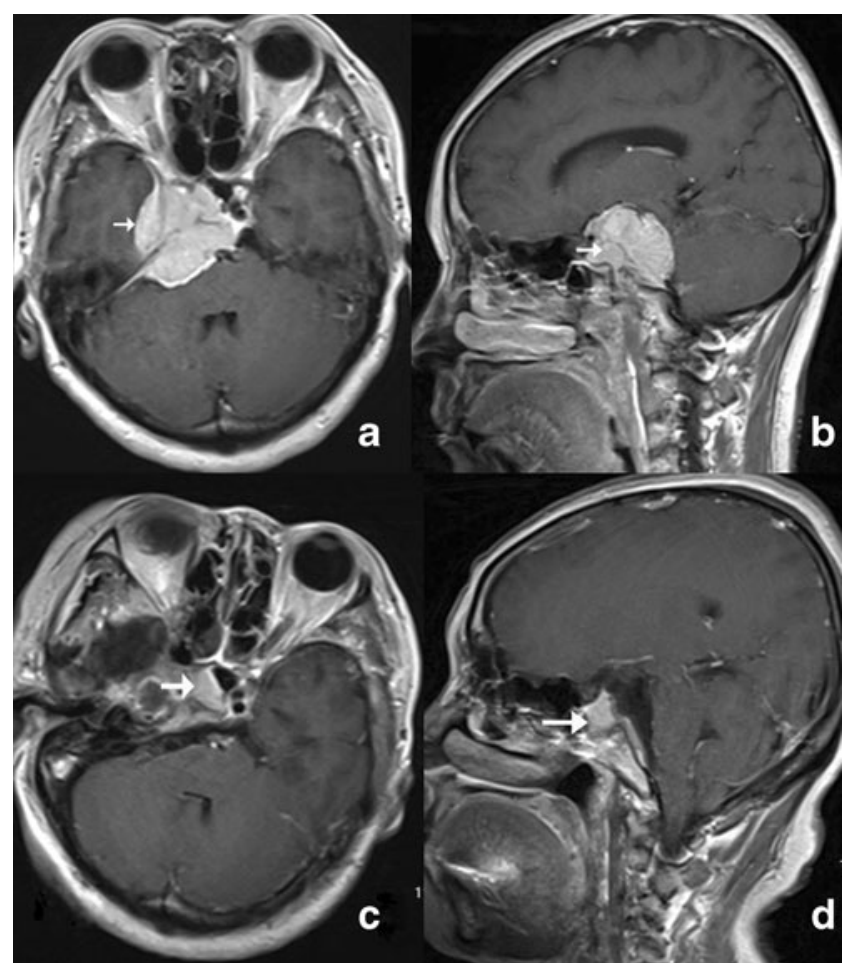

Fig. 1 MRI images of petroclivus meningioma. a, b Preoperative contrast MRI images. There is a tumor significant enhancement (white arrow) located at right petroclivus area and extending into right middle fossa, cavernous sinus, and sphenoid sinus. c, d Postoperative contrast MRI images. The tumor was subtotally removed. The intra-sphenoid sinus component of the tumor appears residually (white arrow)

fascia and the periosteum were left intact. After the skin flap was elevated, the periosteum covering on the zygomatic arch was cut near the temporomandibular joint and was elevated from posterior to anterior. The elevating maneuver was confined to the subperiosteal plane so that the frontotemporal branches of the facial nerve are preserved in the soft tissue flap. The temporalis fascia was opened with a tilted T-shaped incision (Fig. 2b). The temporalis muscle was incised curvely along the temporalis muscle fiber direction (Fig. 2b) starting from the top of mandibular joint and extending to the end of skin incision. Then, the temporalis muscle was retracted bilaterally with a self-retaining retractor. A $4 \times 6-\mathrm{cm}$ bone flap was elevated. The craniotomy was centered above the external auditory canal. Bone was removed to the floor of the middle cranial fossa with ronguers and drill.

After dura was opened and reflected as an inferior base flap over the zygomatic arch, the temporal lobe was elevated gently with a self-retaining retractor. Careful attention was given to the protection of the vein of Labbè and the large draining veins in the base of temporal lobe to prevent postoperative cerebral hemorrhage due to vein occlusion. Hyperventilation, osmotics, and cerebrospinal fluid (CSF) drainage are used when appropriate. The tentorium was transected behind the entrance of trochlear nerve and then 


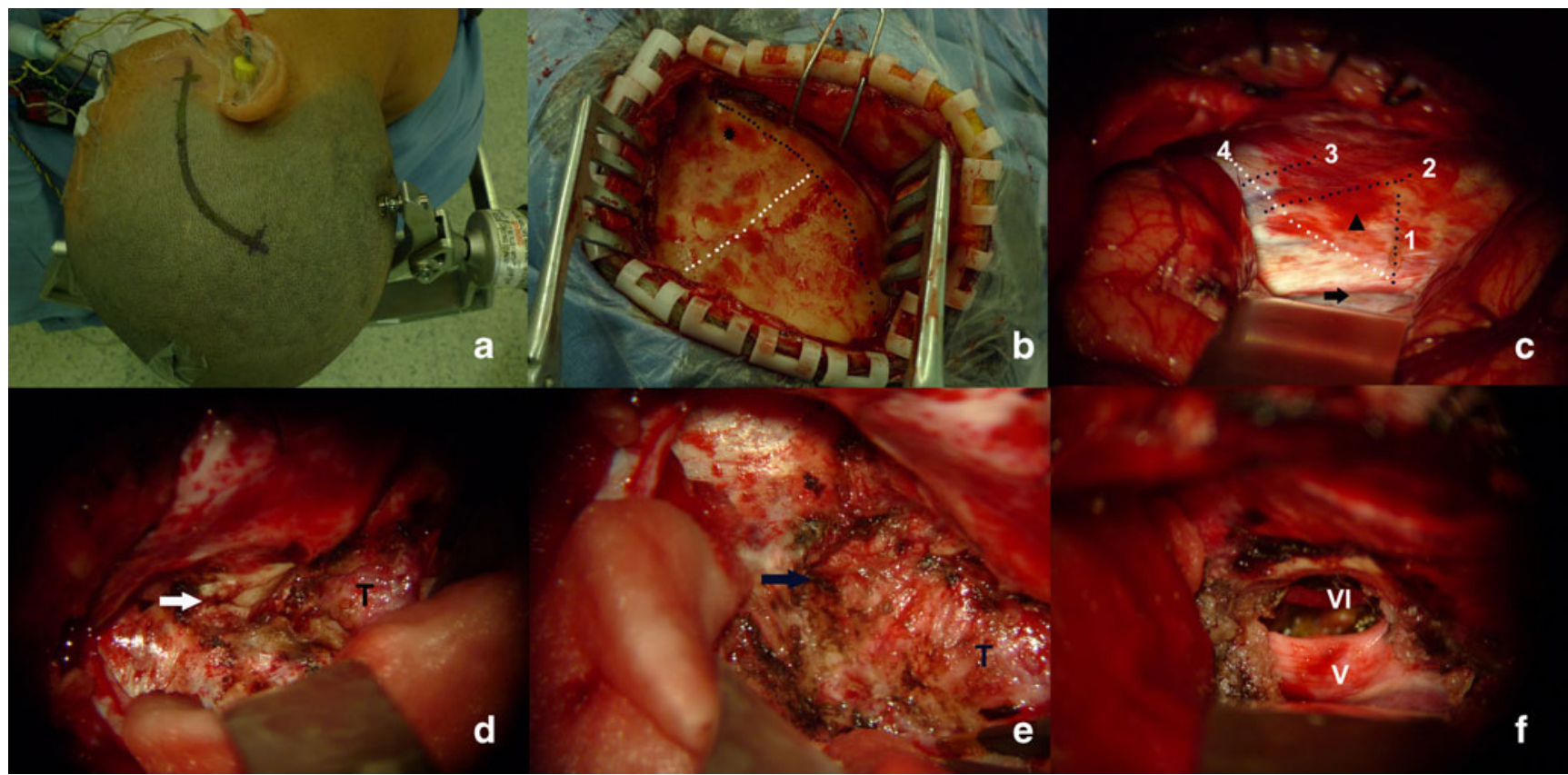

Fig. 2 Operative pictures. a The patient's position and the skin incision. The patient was placed in a lateral decubitus position. The head was fixed in a three-point Mayfield head holder. The curvilinear skin incision was used. b Exposure of temporal bone. The temporalis fascia and muscle were cut in an inferoanterior to superoposterior manner (black dashed line). The temporalis fascia was then incised superoanteriorly (white dashed line). The temporalis fascia and muscle were retracted bilaterally with a self-retaining retractor. The temporal bone and the posterior part of zygomatic arch were exposed (black star). c The transection of tentorium of cerebellum. The tentorium of cerebellum was transected posterior to the entrance of trochlear nerve (black dashed

along the superior petrosal sinus. The dura on the petrous apex was coagulated and incised along the superior petrosal sinus. The superior petrosal sinus was elevated from petrosal bone and then cauterized and divided (Fig. 2c). The dura on the petrous apex was elevated medially until the trigeminal impression was identified. A part of tentorium was removed to enhance the exposure of the posterior fossa (Fig. 2c). The petrous apex bone was thus exposed (Fig. 2d) and the drill beginning internally from the trigeminal impression, usually not exceeding $1.5 \mathrm{~cm}$ laterally, forwardly not exceeding $6 \mathrm{~mm}$ from the posterior edge of the petrous ridge, and not exceeding $8 \mathrm{~mm}$ in depth from the surface of the petrous bone (Fig. 3).

After petrosal apex bone removal (Fig. 2e), Meckel's cavity and the dura in the middle skull base were incised forwardly along the third root of trigeminal nerve. The base of the tumor in CS was often located at the upper dura of the Meckel's cavity and could be dissected safely. The tumor was debulked with an ultrasonic sound absorber. Special care was taken to avoid injury of the trochlear nerve, oculomotor nerve, trigeminal nerve, abducens nerve (Fig. 2f), and basilar artery and its branches. In cases where the tumor was closely adhered to basilar artery or brain stem, a subtotal removal was recommended because complete line 1) and then along the superior petrosal sinus (black dashed line 2). The dura on the petrous apex was coagulated and incised along the superior petrosal sinus (black dashed line 3 ). The superior petrosal sinus was elevated from petrosal bone and then was cauterized and divided (white dashed line 4). A portion of tentorium was removed to enhance the exposure of the posterior fossa (black triangle). d Exposure of the tumor $(T)$ and petrosal apex bone (white arrow). e The petrosal apex bone was removed, and the base of the tumor was exposed (black arrow). f After tumor was resected, the cranial nerves V and VI were intact and could be visualized. $T$ tumor, $V$ cranial nerve $\mathrm{V}$, $V I$ cranial nerve VI

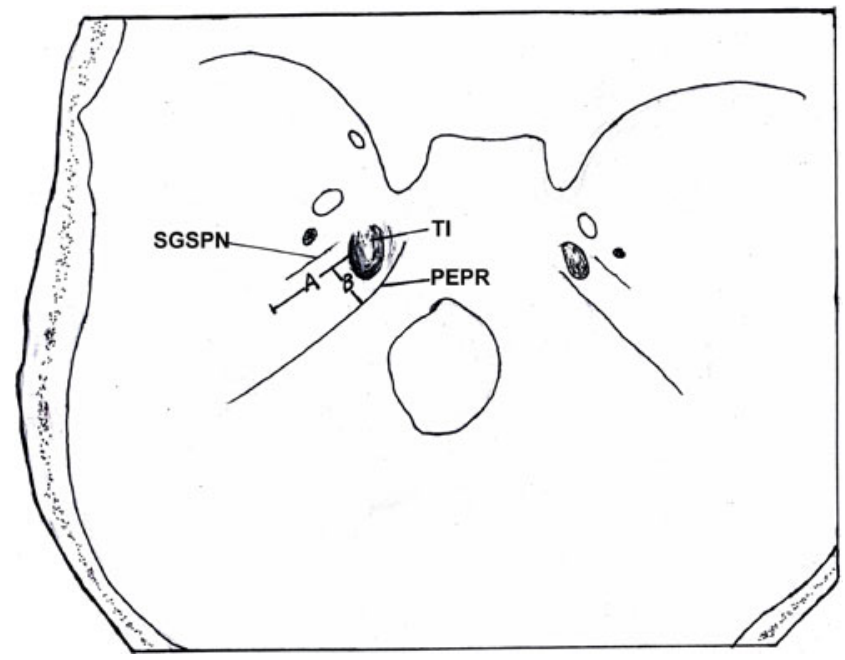

Fig. 3 Illustration showing the drilling extent of petrous apex bone. The trigeminal impression and the posterior edge of petrous ridge were used as landmarks. The drill beginning internally from the trigeminal impression, usually not exceeding $1.5 \mathrm{~cm}$ laterally (line $A$ ) and not exceeding $6 \mathrm{~mm}$ from the posterior edge of the petrous ridge (line $B$ ). $S G S P N$ sulcus of the greater superficial petrosal nerve, $P E P R$ posterior edge of petrous ridge, $T I$ trigeminal impression 
resection of these tumors would cause more serious morbidities, including hemiplegia and coma. Hemorrhage in the Meckel's cavity and the cavernous sinus after removal of the tumor was managed by gelatin sponge tamponade. The drilled petrous apex bone was sealed with bone wax. The dura was closed in a watertight fashion. The bone flap was fixed in place with titanium plate. The wound was closed in layers.

\section{Results}

All 21 cases underwent the modified anterior transpetrous approach. The tumors were totally resected in 12 cases, subtotally in 8 cases, and partially in 1 case. The partially resected case had previously received surgical treatment and $\gamma$-knife therapy. The tumor was of a firm consistency, and the subarachnoid space between the tumor and the brain stem disappeared. Of the nine incompletely removed tumors, two cases received radiosurgery because of the tumor regrowth according to the follow-up MRI. The remaining seven cases were followed up with only close observation. Two patients experienced transient mild hemiplegia and recovered within 2 months after the operation. Three patients developed language disorder after surgery, of which two patients presented with delayed speech and one with incomplete motor aphasia. All the three patients recovered within 2 months after surgery. Other newly developed symptoms and signs, including abducens nerve palsy, oculomotor nerve palsy, and facial numbness, are listed in Table 2. Two patients developed symptoms of meningitis after surgery and were cured after antiinflammatory treatment. Improvement of the preoperative cranial nerve dysfunction is reported in Table 3. The pathological examination reveals a meningioma in all cases. Postoperative thin-slice CT shows the drilling extent of petrous apex bone (Fig. 4). There were no deaths in this series after the operation. All of the 21 patients had a KPS score greater than 80 and were independent in daily life.

Table 2 Postoperative complications and newly developed cranial nerve dysfunction

\begin{tabular}{lll}
\hline $\begin{array}{l}\text { Postoperative } \\
\text { complications }\end{array}$ & $\begin{array}{l}\text { Early postoperative } \\
\text { phase }\end{array}$ & $\begin{array}{l}\text { At the end of } \\
\text { follow-up }\end{array}$ \\
\hline Hemiplegia & 2 & 0 \\
Aphasia & 3 & 0 \\
Meningitis & 2 & 0 \\
Hearing impairment & 1 & 1 \\
Oculomotor nerve palsy & 3 & 0 \\
Facial palsy & 1 & 0 \\
Abducens nerve palsy & 6 & 0 \\
Facial numbness & 6 & 4 \\
\hline
\end{tabular}

Table 3 Improvement of preoperative cranial nerve dysfunction

\begin{tabular}{lll}
\hline $\begin{array}{l}\text { Cranial nerve } \\
\text { dysfunction }\end{array}$ & $\begin{array}{l}\text { Preoperative } \\
\text { occurrence }(n)\end{array}$ & $\begin{array}{l}\text { Postoperative } \\
\text { improvement }(n)\end{array}$ \\
\hline Optic nerve & 3 & 3 \\
Oculomotor nerve & 1 & 1 \\
Facial numbness & 13 & 2 \\
Facial pain & 7 & 7 \\
Abducens nerve & 4 & 4 \\
Facial nerve & 1 & 1 \\
Auditory nerve & 3 & 1 \\
Caudal cranial nerves & 2 & 2 \\
\hline
\end{tabular}

\section{Discussion}

As large and giant PCMs often involve critical neurovascular structures and brain stem, the surgical outcomes of PCMs were once unsatisfactory with only very limited tumor removal and/or high complication rates $[5,21]$. As extensive skull base microanatomic dissection work was initiated in the late 1980s, various skull base approaches including presigmoid transpetrosal approach, ATPA, and so forth were developed and applied for the treatment of PCMs [1, 2, 6, 28].

The presigmoid transpetrosal approach can expose the tumor sufficiently and allow tumor removal via multiple angles to improve the total resection rate [7, 8, 20, 22, 27]. It was once considered as the first option for PCMs and was often followed by an overaggressive attempt of complete tumor removal, even in cases of significant tumor-brain stem adherence and vessel and cranial nerve encasement. However, the increasing use of this complicated skull base approaches also results in a consequent increase of approachrelated complications including high risk of infection, hearing impairment, and high CSF leak risk [32]. Most PCMs are slowly growing benign neoplasms [31]. Stereotactic radiosurgery has been proved to be an effective treatment modality as a first-line treatment for small PCMs or as an adjuvant to surgical resection $[10,13,25]$. Preservation of the quality of life in the PCM patients has become the top treatment priority. Therefore, many neurosurgeons have moved away from performing complex transpetrosal approaches to treat these challenging lesions. Less aggressive cranial base approaches have been used as alternative approaches for PCMs, including the traditional retrosigmoid approach, the subtemporal approach, the combined supra-infratentorial keyhole approach, the anterior transpetrosal approach, and the frontorbitozygomatic approach [3, 12, 18, 23, 26, 32, 33].

The ATPA was first described by Bochenek and Kukwa [4]. Kawase and colleagues modified this approach in order to expose the lower basilar artery, petroclival tumors, and brain stem lesions [14-17]. This approach consisted of drilling of the petrous apex extradurally through the middle 
Fig. 4 Postoperative thin-slice $\mathrm{CT}$ of the presented case. The postoperative thin-slice CT shows the drilling range of petrous apex bone (white arrow)

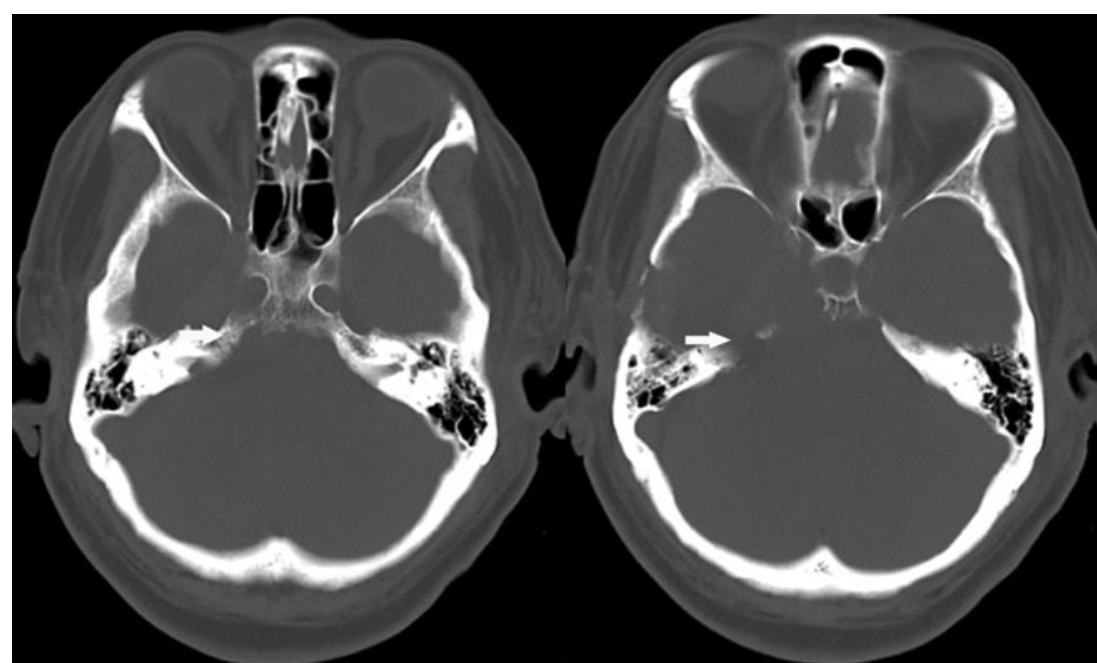

fossa to expose the cerebellopontine angle and cutting the tentorium to better visualize the brain stem and basilar artery. It also had the added benefit of allowing improved visualization of the clivus across the midline. However, there are several disadvantages to perform the anterior petrosal approach. First, it requires advanced anatomic dissection training. Second, extradural dissection is often associated with troublesome extradural bleeding [9]. Third, the dura is usually adhered firmly to the petrosquamousal suture, and the greater superficial petrosal nerve is always medial to this suture. Malmanipulation of greater superficial petrosal nerve would result in facial nerve palsy [11]. Fourth, widely elevating the dura of middle fossa could increase the postoperative CSF leakage rate. Fifth, this approach requires another operation to harvest fat tissue to obliterate the bone defect $[9,11]$.

In the last 2 years, a modified ATPA was developed and used in the surgical treatment of 21 large and giant PCMs in our department. Compared with the conventional ATPA, our modifications are as follows:

First, a curvilinear incision was used in our cases. The curvilinear incision in the ATPA for the surgery of PCMs was first described by Hitselberger and his colleagues. However, the incision used by Hitselberger begins closely to the tragal notch which limits the anterior exposure of the middle fossa and cavernous sinus. In our surgeries, the curvilinear skin incision was made at the midpoint of zygomatic arc, and the temporalis fascia and the periosteum covering on the zygomatic arch were left intact. After the skin flap was elevated, the periosteum covering on the zygomatic arch was cut near the temporomandibular joint and was elevated from posterior to anterior. The elevating maneuver was confined to the subperiosteal plane so that the frontotemporal branches of the facial nerve are preserved in the periosteum flap. Therefore, the curvilinear skin incision used in our cases is more anteriorly and lower compared to that reported by Hitselberger and could obtain better visualization of the anterior part of middle fossa and cavernous sinus.
Second, the temporalis muscle was incised along the direction of temporalis muscle fibers and was retracted bilaterally in our cases. As a result, much lower exposure of the bottom of middle fossa and less temporalis muscle trauma were achieved. In the traditional ATPA, the temporalis muscle was generally cut and reflected inferiorly which would obstacle the bottom exposure of middle fossa [9, 11, 14-17]. Although an additional zygomatic osteotomy could provide a sufficient exposure to the middle fossa bottom [9], a zygomatic osteotomy and opening of temporomandibular joint capsule would result in chewing dysfunction. As the temporal muscle is less affected, no significant postoperative temporal muscle atrophy occurred in our series.

Third, drilling of the petrous apex bone was performed subdurally. This method is commonly used and can also be performed more easily by most neurosurgeons - with limited dissection training course in the anatomy lab. Although drilling the petrous apex bone subdurally was also mentioned in several reports $[9,32]$, the landmark and the extent of bone drilling were not specified in these reports. In our cases, the trigeminal impression and posterior edge of petrous ridge were used as landmarks (Fig. 3). A $1.5 \times 0.6-\mathrm{cm}$ area of petrous apex bone drilling was sufficient to expose the base of the petroclivus meningiomas. Simultaneously, the internal carotid artery and the greater superficial petrosal nerve were not exposed and were thus protected from damage. In the traditional ATPA, a $2 \times 1-\mathrm{cm}$ area of petrous apex bone was drilled extradurally, and the horizontal segment of the petrous internal carotid artery might have been exposed and damaged [11]. The drilled petrous apex bone was sealed with bone wax in this series, and fortunately, no postoperative CSF leak occurred. This may be due to the case number is small. Using temporalis fascia and fibrin glue to seal, the drilled petrous apex bone might further reduce the risk of CSF leak. In the present series, postoperative hearing loss of the affected side was observed in one case, and transient mild facial palsy occurred in one case. 
Nevertheless, there are also several concerns regarding our modified procedure as compared with the conventional ATPA: First, earlier subdural manipulation may increase the risk of temporal lobe contusion. Second, the draining veins of the temporal lobe might have a higher risk of damage during this procedure. In order to reduce the damage to the temporal lobe, it is vital to decrease the intracranial pressure at the beginning of the operation to reduce the damage of temporal lobe. Several methods, including decreasing the airway pressure below $16 \mathrm{~cm}$ during surgery and using of $250 \mathrm{ml} 20 \%$ mannitol, were used and decreased intracranial pressure significantly during the operation. In patients with a giant tumor, preoperative cerebrospinal fluid drainage was used to decrease the intracranial pressure. The damage of draining vein is often associated with high risk of postoperative hemorrhage and significant edema in temporal lobe. Therefore, all of the draining veins of the temporal lobe should be protected as much as possible. If the subtemporal draining veins enter into the middle fossa dura, the dura should be cut along the course of vein to obtain a sufficient mobilization of the vein. Following these principles, only one elderly patient in our series presented with mild radiographic brain contusion after surgery and recovered very well. However, the draining veins could not be preserved easily. The brain tissue of senior patients is more vulnerable to the retraction. If there are large draining veins on the base of temporal lobe on the preoperative MRV images or the patient is in advanced age, other approaches such as retrosigmoid approach may be a better selection.

In large tumors, the brain stem was usually displaced posteriorly and the surgical corridor to the tumor is usually made by the tumor itself. The retrosigmoid approach is a fast, easy, and straightforward approach and the tumor could be removed with almost no retraction of the brain stem. In those rather small PCMs, the brain stem was not displaced and the tumors are more difficult to approach via posterior approaches. In PCMs with a firm consistency or contralateral cranial nerve, involving the ATPA could provide a better view and a wider surgical field and provide a better chance to preserve the function of the brain stem and the contralateral cranial nerve. Therefore, the true advantage of the anterior petrosal approach is in the management of those rather small, a firm consistency, and contralateral cranial nerve involving PCMs.

Compared with the presigmoid approach, our modified ATPA could obtain the gross total and subtotal tumor resection rate similar to that of presigmoid approach in a selected group of PCMs, and it could also save $2-3 \mathrm{~h}$ in opening and closing procedure time during operation without the risk of causing sigmoid sinus injury. However, as the modified approach is unable to expose the lateral aspects of the IAM, it is still necessary to use the presigmoid transpetrosal approach or the combined suboccipital retrosigmoid approach when the tumor extend lateral to the IAM.

Although many neurosurgeons suggest that the tumor in cavernous sinus should be left behind to avoid cranial nerve dysfunction, according to our experience, the base of most of the tumors in cavernous sinus is located at the upper wall of Meckel's cavity and could be easily dissected. As the tumor inside the cavernous sinus is usually not significantly adhered to the surrounding tissues, it could usually be removed safely. To avoid damage to the surrounding nerves, hemostasis of the cavernous sinus is usually achieved by gelatin sponge tamponade rather by coagulation. If the tumor in cavernous sinus was of a firm consistency, tumor would adhere to the surrounding cranial nerves and vessels firmly and a subtotal or partial removal would be recommended.

Postoperative hemorrhage in the brain stem is likely to occur in patients that have T2-weighted MR imaging showing severe brain stem edema. We have several such cases in our earlier cases. Later, dexamethasone (10 $\mathrm{mg}$, twice/day) or methylprednisolone ( $80 \mathrm{mg}$, twice/day) was given intravenously for 2 weeks and no postoperative hemorrhage occurred in brain stem again even in cases where cerebral edema was not relieved significantly on MR images. The presence of brain stem edema on T2-weighted MR images and blood supply to the tumor from the brain stem on the contrast enhancement of MRI indicated a disruption of the blood-brain barrier and invasion or adherence of the tumor to the brain stem [29]. If the tumors invade or adhere to the brain stem or the basilar artery and other important vessels are encased by the tumor, a subtotal resection was recommended to avoid the catastrophic complications. The residual tumor can be followed up regularly by MRI. Should tumor growth be detected, $\gamma$-knife therapy could be considered.

\section{Conclusion}

In summary, the modified ATPA has the following advantages: (1) It could provide sufficient exposure for the PCMs located in the middle and upper petroclivus and the medial side of the IAM. (2) It provides more sufficient exposure of the bottom of middle fossa compared to the conventional ATPA. (3) Neurosurgeons are more familiar with the procedure, and there is less temporal muscle atrophy and less risk of postoperative facial palsy and CSF leak compared with the conventional ATPA. (4) And no other operation is needed to obtain the fat tissue to repair the skull base.

Conflict of Interest The authors report no conflict of interest concerning the materials and methods used in this study or the findings specified in this paper. 
Open Access This article is distributed under the terms of the Creative Commons Attribution License which permits any use, distribution, and reproduction in any medium, provided the original author(s) and the source are credited.

\section{References}

1. Al-Mefty O, Fox JL, Smith RR (1988) Petrosal approach for petroclival meningiomas. Neurosurgery 22:510-517

2. Al-Mefty O, Ayoubi S, Smith RR (1991) The petrosal approach: indications, technique, and results. Acta Neurochir Suppl (Wien) 53:166-170

3. Bambakidis NC, Kakarla UK, Kim LJ, Nakaji P, Porter RW, Daspit CP, Spetzler RF (2007) Evolution of surgical approaches in the treatment of petroclival meningiomas: a retrospective review. Neurosurgery 61([ONS Suppl 2]):ONS202-ONS211

4. Bochenek J, Kukwa A (1975) An extended approach through the middle cranial fossa to the internal auditory meatus and the cerebellopontine angle. Acta Otolaryngol 79:410-414

5. Bricolo AP, Turazzi S, Talacchi A, Cristofori L (1992) Microsurgical removal of petroclival meningiomas: a report of 33 patients. Neurosurgery $31: 813-828$

6. Canalis RF, Black K, Martin N, Becker D (1991) Extended retrolabyrinthine transtentorial approach to petroclival lesions. Laryngoscope 101:6-13

7. Cho CW, Al-Mefty O (2002) Combined petrosal approach to petroclival meningiomas. Neurosurgery 51:708-716

8. Couldwell WT, Fukushima T, Giannotta SL, Weiss MH (1996) Petroclival meningiomas: surgical experience in 109 cases. J Neurosurg 84:20-28

9. Harshiv GR, Sekhar LN (1992) The subtemporal, transcavernous, anterior transpetrosal approach to the upper brain stem and clivus. J Neurosurg 77:709-717

10. Henzel M, Gross MW, Hamm K, Surber G, Kleinert G, Failing T, Strassmann G, Engenhart-Cabillic R (2006) Significant tumor volume reduction of meningiomas after stereotactic radiotherapy: results of prospective and multicenter study. Neurosurgery 59(6):1188-1194

11. Hitselberger WE, Horn KL, Hankinson H, Brackmann DE, House WF (1993) The middle fossa transpetrous approach for petroclival meningiomas. Skull Base Surgery 3(3):133-135

12. Ichimura S, Kawase T, Onozuka S, Yoshida K, Ohira T (2008) Four subtypes of petroclival meningiomas: differences in symptoms and operative findings using the anterior transpetrosal approach. Acta Neurochir (Wien) 150:637-645

13. Iwai Y, Yamanaka K, Nakajima H (2001) Two-staged gamma knife radiosurgery for the treatment of large petroclival and cavernous sinus meningiomas. Surg Neurol 56:308-314

14. Jung HW, Yoo H, Paek SH, Choi KS (2000) Long-term outcome and growth rate of subtotally resected petroclival meningiomas: experience with 38 cases. Neurosurgery 46:567-574, discussion 574-565

15. Kawase T, Shiobara R, Toya S (1991) Anterior transpetrosaltranstentorial approach for sphenopetroclival meningiomas: surgical method and results in 10 patients. Neurosurgery 28:869-875

16. Kawase T, Shiobara R, Toya S (1994) Middle fossa transpetrosaltranstentorial approaches for petroclival meningiomas. Selective pyramid resection and radicality. Acta Neurochir (Wien) 129:113-120

17. Kawase T, Toya S, Shiobara R, Mine T (1985) Transpetrosal approach for aneurysms of the lower basilar artery. J Neurosurg 63:857-861

18. Li PL, Mao Y, Zhu W, Zhao NQ, Zhao Y, Chen L (2010) Surgical strategies for petroclival meningioma in 57 patients. Chin Med J (Eng) 123(20):2865-2873

19. Little KM, Friedman AH, Sampson JH, Wanibuchi M, Fukushima $\mathrm{T}$ (2005) Surgical management of petroclival meningiomas: defining resection goals based on risk of neurological morbidity and tumour recurrence rates in 137 patients. Neurosurgery 56:546-559

20. Mathiesen T, Gerlich A, Kihlstrom L, Svensson M, Bagger-Sjoback D (2007) Effects of using combined transpetrosal surgical approaches to treat petroclival meningiomas. Neurosurgery 60:982-991

21. Mayberg MR, Symon L (1986) Meningiomas of the clivus and apical petrous bone. Report of 35 cases. J Neurosurg 65:160-167

22. Natarajan SK, Sekhar LN, Schessel D, Morita A (2007) Petroclival meningiomas: multimodality treatment and outcomes at long-term follow-up. Neurosurgery 60:965-981

23. François P, Ismail MB, Hamel O, Bataille B, Jan M, Velut S (2010) Anterior transpetrosal and subtemporal transtentorial approaches for pontine cavernomas. Acta Neurochir 152:1321-1329

24. Ramina R, Neto MC, Fernandes YB, Silva EB, Mattei TA, Aguiar PH (2008) Surgical removal of small petroclival meningiomas. Acta Neurochir (Wien) 150:431-439

25. Roche PH, Pellet W, Fuentes S, Thomassin JM, Regis J (2003) Gamma knife radiosurgical management of petroclival meningiomas results and indications. Acta Neurochir (Wien) 145:883-888

26. Seifert V (2010) Clinical management of petroclival meningiomas and the eternal quest for preservation of quality of life. Acta Neurochir 152:1099-1116

27. Seifert V, Raabe A, Zimmermann M (2003) Conservative (labyrinth-preserving) transpetrosal approach to the clivus and petroclival region - indications, complications, results and lessons learned. Acta Neurochir (Wien) 145:631-642

28. Sekhar LN, Wright DC, Richardson R, Monacci W (1996) Petroclival and foramen magnum meningiomas: surgical approaches and pitfalls. J Neurooncol 29:249-259

29. Simis A, Pires de Aguiar PH, Leite CC, Santana PA Jr, Rosemberg S, Teixeira MJ (2008) Peritumoral brain edema in benign meningiomas: correlation with clinical, radiologic, and surgical factors and possible role on recurrence. Surg Neurol 70:471-477

30. Tahara A, De Santana PA Jr, Calfat Maldaun MV, Panagopoulos AT, da Silva AN, Zicarelli CA, Pires de Aguiar PH (2009) Petroclival meningiomas: surgical management and common complications. J Clin Neurosci 16:655-659

31. Van Havenbergh T, Carvalho G, Tatagiba M, Plets C, Samii M (2003) Natural history of petroclival meningiomas. Neurosurgery 52:55-62

32. Yang J, Ma SC, Fang T, Qi JF, Hu YS, Yu CJ (2011) Subtemporal transpetrosal apex approach: study on its use in large and giant petroclival meningiomas. Chin Med J (Eng) 124(1):49-55

33. Zhu W, Mao Y, Zhou LF, Zhang R, Chen L (2006) Keyhole approach surgery for petroclival meningioma. Chin Med J (Eng) 119(16):1339-1342

This research was approved by the institutional review board of the Beijing Tiantan Hospital and was not funded.

\section{Comments}

Feng $\mathrm{Xu}$ and Nicholas C. Bambakidis, Cleveland, USA

The authors described a modified anterior transpetrous approach for the surgical resection of petroclival meningiomas. They transected the tentorium and performed intradural petrous apex resection. The authors concluded that the modified anterior transpetrous approach is an efficient treatment alternative for large or giant petroclival meningiomas located at the medial and superior internal auditory canal (IAC) with relatively low risk of complications.

Anterior transpetrosal approach is best suited for small petroclival meningiomas medial to IAC without extensive involvement with the posterior fossa. Extradural anterior petrosectomy is limited by the 
greater superficial petrosal nerve (GSPN) laterally, petrous ridge medially, mandibular nerve anteriorly, and the IAC posteriorly. There are potential surgical risks and complications. Firstly, traction injury of GSPN can lead to facial nerve palsy. Secondly, there is the not insignificant risk of postoperative CSF leakage as a result of an inability to achieve adequate dural closure at the skull base. Intradural anterior petrosectomy here is an alternative treatment option for removal of petroclival meningiomas. However, this modified approach also requires retraction of the temporal lobe and draining veins. The surgeon would better evaluate the venous drainage pattern through magnetic resonance venography before operation. The temporal lobe and draining veins should be protected as far as possible during operation.

The modified approach is preferred for lesions which span both middle and posterior cranial fossae and located medial to the IAC. However, this approach is limited in its ability to expose lesions that are located laterally to IAC. If lesions extended forward into the posterior fossa or lateral to IAC, a posterior transpetrosal approach or retrosigmoid craniotomy can be used. In recent decades, we have shift from performing aggressive transpetrosal approach to conventional retrosigmoid approach, particularly for lesions lateral to the IAC or without a significant supratentorial extension. For large legions such as those discussed in this paper, we continue to utilize an extradural transpetrous approach. The intradural modification described in this article seems to be a reasonable alternative in dealing with these challenging lesions.

Takeshi Mikami, Sapporo, Japan

In this paper, the authors introduced an intradural drilling technique for anterior petrosectomy in patients with petroclival meningiomas. Intradural drilling makes it possible to drill away only the required field; this technique is widely used for removal of the anterior clinoid process or jugular tuberculum. I agree that these minimal exposures are reasonable and could help avoid extradural complications. The authors have presented excellent results. The following are some notes.

First, careful and meticulous drilling is required. The vein of Labbé exits to the front in the surgical field, and management of this vein is important. Second, in order to avoid catastrophic complications, the authors recommended a subtotal resection in patients in which the tumor has invaded the brain stem or vascular components. However, wider exposure with extradural drilling or a presigmoid approach may improve recognition in these adhered cases. As for the reconstruction, a free graft without vascular supply must be used for reconstruction of the petrous apex in the authors' modified approach because of the minimal skin and temporal muscle incision. The authors reported that no CSF leaks were experienced in this series. Indeed, the temporal fascia and fibrin glue can adequately seal the petrous apex if the drilled space is limited. This is because sinus pneumatization does not usually reach this part. However, preoperative recognition of pneumatization using thinslice computed tomography should be considered important.

Although it is a beneficial technique that should be introduced widely through publication, it is, unfortunately, not likely that this report constitutes a new strategy for this surgical procedure. In addition, this method does not reduce all of the surgical risks. An in-depth anatomical understanding of the temporal bone remains necessary for surgery on petroclival meningiomas.

\section{Florian Roser, Tübingen, Germany}

The authors report about a technical nuance in the surgical management of petroclival meningiomas. They advocate an intradural subtemporal approach to the petrous apex and demonstrate favorable results. Although they present mainly "giant" petroclival meningiomas, I think that the true advantage of the anterior petrosal approach is in the management of rather small petroclival meningiomas, as these do not displace the brain stem and are more difficult to approach via posterior approaches. Moreover, petroclival meningiomas most often show a cranio-caudal extension along the skull base. In the presented approach, the visibility of caudal tumor parts at the level of the jugular foramen is out of reach as well as rostral parts of tumors at suprasellar levels.

In true giant petroclival meningiomas, where the avenue to the tumor is made by the tumor itself and almost no retraction of the brain stem is necessary, the simple retrosigmoid approach is fast, easy, and straightforward. Moreover, the intradural manipulation of Labbé's vein in the discussed approach is more dangerous than in the extradural Kawase approach. The fact that the authors did see complications in only one patient does not mean it is safe. Altogether, this is a nice extension in the armentarium of a skull base surgeon, but the indication for this approach should be tailored and individualized.

\section{Samii, V. Gerganov, Hannover, Germany}

The authors presented their experience with a modification of the anterior transpetrous approach to the petroclival area, Meckel's cave, and medial cerebellopontine angle. Using this approach, in a series of 21 patients with large and giant petroclival meningiomas, they achieved good results in terms of completeness of resection and morbidity rate, which is certainly a merit of their surgical proficiency.

Over the last decades, the concept of surgery of these challenging tumors advanced considerably. Currently, we know that extensive exposure of skull base tumors with all surrounding neurovascular structures is not a prerequisite for safe and radical removal. On the contrary, this may increase the risk of complications. In case of petroclival tumors, we favor the retrosigmoid approach with intradural resection of the suprameatal tubercle - the so-called retrosigmoid suprameatal (Samii's) approach [1, 2]. Drilling of the suprameatal tubercle provides sufficient access to the petroclival area, Meckel's cave, and even the posterior part of the cavernous sinus [3]. Further exposure could be gained, if necessary, by additional incision and retraction of the tentorium. Rigid endoscopes allow for detecting hidden parts of the tumor and even for resecting them using appropriate angled instruments that are useful complimentary tools.

\section{References}

1. Samii M, Tatagiba M, Carvalho GA (1999) Resection of large petroclival meningiomas by the simple retrosigmoid route. J Clin Neurosci 6(1):27-30.

2. Samii M, Tatagiba M, Carvalho GA (2000) Retrosigmoid intradural suprameatal approach to Meckel's cave and the middle fossa: surgical technique and outcome. J Neurosurg 92(2):235-241.

3. Samii M, Gerganov VM (2008) Surgery of extra-axial tumors of the cerebral base. Neurosurgery 62 (6 Suppl 3):1153-1166. 